\title{
Up-regulation of the anti-inflammatory adipokine adiponectin in acute liver failure in mice
}

\author{
Anna Maria Wolf ${ }^{1}$, Dominik Wolf ${ }^{2}$, Matias A. Avila ${ }^{3}$, Alexander R. Moschen ${ }^{1}$, \\ Carmen Berasain ${ }^{3}$, Barbara Enrich ${ }^{1}$, Holger Rumpold ${ }^{2}$, Herbert Tilg ${ }^{1, *}$ \\ ${ }^{1}$ Division of Gastroenterology and Hepatology, Department of Internal Medicine, Innsbruck Medical University, Innsbruck, Austria \\ ${ }^{2}$ Division of Hematology and Oncology, Department of Internal Medicine, Innsbruck Medical University, Innsbruck, Austria \\ ${ }^{3}$ Division of Hepatology and Gene Therapy, CIMA, Universidad de Navarra, Pamplona, Spain
}

Background/Aims: Recent reports suggest that the adipose tissue and adipokines are potent modulators of inflammation. However, there is only scarce knowledge on the functional role and regulation of endogenous adiponectin in non-fat tissues such as the liver under conditions of acute inflammation.

Methods: In the present study, we investigated adiponectin expression in healthy murine liver tissue and under inflammatory conditions in vivo.

Results: Adiponectin mRNA was readily detectable in healthy liver tissue and further increased in ConA-mediated acute liver failure. Adiponectin protein expression was mainly found in hepatic endothelial cells. In vitro adiponectin mRNA expression was detectable in several cell types, including primary hepatic sinusoidal endothelial cells, stellate cells, and macrophages. Mice pretreated with adiponectin before ConA administration developed reduced hepatic injury as shown by decreased release of transaminases and reduced hepatocellular apoptotis. Of note, TNF- $\alpha$ levels were not affected by adiponectin, whereas IL-10 production was increased. Neutralisation of IL-10 diminished the protective effect of adiponectin.

Conclusions: Adiponectin expression is up-regulated in ConA-mediated acute liver failure. Therefore, adiponectin might play a role in the control and limitation of inflammation in the liver. Moreover, our data suggest a role for IL-10 in adiponectin-mediated hepatoprotection.

(C) 2005 European Association for the Study of the Liver. Published by Elsevier B.V. All rights reserved.

Keywords: Adiponectin; ConA; Inflammation; Acute liver failure; Cytokines

\section{Introduction}

Only recently, with the discovery of a variety of adipocyte-derived cytokines, so called 'adipokines', the adipose tissue has gained a role as an important endocrine organ. Adiponectin has attracted much interest as it represents the most abundant adipocyte-derived protein reaching high plasma levels in both humans and rodents [1].

Received 10 May 2005; received in revised form 24 August 2005; accepted 31 August 2005; available online 23 September 2005

* Corresponding author. Address: Division of Gastroenterology and Hepatology, Department of Medicine, University Hospital Innsbruck, Anichstr 35, 6020 Innsbruck, Austria. Tel.: + 43512504 0; fax: +43 512 50423391.

E-mail address: herbert.tilg@uibk.ac.at (H. Tilg).
Adiponectin has been shown to elicit a broad range of biological effects such as insulin-sensitising and antiatherogenic actions [2].

Recent reports pointed out to a possible role of adipose tissue and adipokines as potent regulators of inflammatory processes. We and others recently demonstrated potent antiinflammatory properties of adiponectin [3,4]. Apart from downregulation of the synthesis of the pro-inflammatory cytokine TNF- $\alpha$, adiponectin leads to an increased production of the anti-inflammatory mediators IL-10 and IL-1RA in human myeloid cells [3]. Adiponectin seems to be primarily expressed by adipocytes. Thus, regulation of adiponectin expression has been almost exclusively studied in adipocytes. However, recent reports showed that in human myotubes expression of adiponectin can be induced 
upon stimulation with pro-inflammatory stimuli such as TNF- $\alpha$ or LPS $[5,6]$. This observation might describe a negative feedback-loop mediated via adiponectin and fits into the concept that adiponectin plays an anti-inflammatory role in inflammation. However, there is only scarce knowledge on the functional role and regulation of endogenous adiponectin and its receptors in non-fat tissues such as the liver under conditions of acute inflammation.

Therefore, the aim of the present work was to (I) examine whether adiponectin could be detected in normal healthy liver tissue, (II) to further characterize adiponectin producing cell types and (III) to find out, if adiponectin exerts hepatoprotective effects in a model of acute liver failure.

\section{Materials and methods}

\subsection{Animals}

Six to 8-week-old female BALB/c mice were obtained from Harlan (Borchen, Germany) and were maintained under controlled conditions ( $22{ }^{\circ} \mathrm{C}, 55 \%$ humidity, 12-h dark/light rhythm), having free access to standard chow and water. All animals received humane care according to the legal requirements in Austria.

\subsection{Animal treatments}

All reagents were injected in a total volume of $200 \mu$ per mouse for intravenous injections (i.v.) and $100 \mu \mathrm{l}$ per mouse for intraperitoneal (i.p.) injections and were dissolved in pyrogen-free saline. Where indicated either $6 \mathrm{mg} / \mathrm{kg}$ murine Adiponectin (R\&D systems) or saline were i.p. injected $12 \mathrm{~h}$ before treatment with $15 \mathrm{mg} / \mathrm{kg}$ Con A (Sigma, Vienna, Austria) i.v. To investigate the role of IL-10 in adiponectin induced hepatoprotection, either $3 \mathrm{mg}$ of neutralizing IL-10 antibody per mouse (Immunotools, Friesoythe, Germany) or solvent was administrated $30 \mathrm{~min}$ before adiponectin injection.

\subsection{Sampling of material}

For determination of circulating cytokine levels, blood samples were taken from the tail-vein $2 \mathrm{~h}$ after challenge. Eight hours after ConAtreatment, mice were lethally anesthetized with $50 \mathrm{mg} / \mathrm{kg}$ Vetanarcol (Intervet, Austria) i.v., containing a dose of $15 \mathrm{mg} / \mathrm{kg}$ heparin. Blood was withdrawn by cardiac puncture, immediately centrifuged and plasma samples were stored at $-20{ }^{\circ} \mathrm{C}$. The livers were removed and one part was frozen in liquid nitrogen for mRNA preparation, another part was embedded in Tissue-TEK and stored at $-20^{\circ} \mathrm{C}$ until staining. Primary macrophages were magnetically selected from minced liver tissue by CD11b beads (Miltenyi Biotec, Bergisch Gladbach, Germany).

\subsection{Primary liver cells}

Primary hepatocytes, hepatic stellate cells (HSC) and hepatic sinusoidal endothelial cells (HSEC) were purchased from Pharmakine (Bilbao, Spain).

\subsection{Cytokine determination by ELISA}

Detection of TNF- $\alpha$, IL-6 and IL-10 in plasma samples was performed strictly according to the manufacturer's instructions using mouse specific monoclonal antibody $(\mathrm{mAb})$ pairs purchased from BD Pharmingen (San Diego, CA).

\subsection{Cytokine determination by cytometric bead array (CBA)}

TNF- $\alpha$, IL-6, and IL-10 in plasma samples were determined using the mouse inflammation kit CBA purchased from BD Pharmingen (San Diego, CA). Analyses were performed strictly according to the manufacturer's instructions.

\subsection{Analysis of liver enzymes}

Liver injury was quantified by measurement of plasma enzyme activities of ALT and AST using an automated procedure.

\subsection{Real-time RT-PCR for $T N F-\alpha, I L-10$, adiponectin, AdipoRl and AdipoR2 mRNA}

Total RNA was prepared from liver tissue using Trizol reagent (Invitrogen, Paisley, UK). One microgram of RNA was employed for synthesis of cDNA using random hexamers (Roche, Mannheim, Germany) and MMLV reverse transcriptase (GIBCO, Gaithersburg, MD) strictly according to the manufacturer's instructions. Primers and TaqMan ${ }^{\circledR}$ Probes specific for TNF- $\alpha$ and IL-10 were obtained from TaqMan ${ }^{\circledR}$ Pre-Developed Assay Reagents (Applied Biosystems, Foster City, CA). For adiponectin, AdipoR1 and AdipoR2 primers and probes were as follows: 5'-CCGGAACCCCTGGCAG-3', 5'-CCGGAACCCCTGGCAG-3', FAM-AAAGGAGAGC CTGGAGAAGCCGCTT-TAMRA, 5'CACTTCCCATGTGCATTCCC-3', 5'-CCCACG TCCTATCTGTGAAGG-3', FAM-ACCTCGGCAAGGCAACTGCTGC-TAMRA, and 5'-AGGAG TGTTCGTGGGCTTAGG-3', 5'-CAGCCTTCAGGAACCCTT CTG-3', FAM-TGAGTGGAATCATCCCTACCTTGCATTATGTC-TAMRA. For endogenous control, the level of GUS expression in each sample was assayed using mouse GUS Pre-developed TaqMan ${ }^{\circledR}$ Control Reagents (Applied Biosystems, Foster City, CA). Quantitative realtime RT-PCR was performed using the ABI PRISM 7700 Sequence Detection System (Applied Biosystems, Foster City, CA). Real-time PCR efficiencies were acquired by amplification of a standardized dilution series of subcutaneous fat (adiponectin), muscle (adipoR1), or liver (adipoR2) cDNA from a Con A-challenged control mouse. The respective cDNAs were further used as controls and arbitrarily set as 1 .

\subsection{DNA fragmentation}

In situ detection of DNA fragments was performed using a terminal deoxynucleotidyl transferase-mediated deoxyuridine triphosphate nick-end labeling (TUNEL) test (Roche, Mannheim, Germany).

\subsubsection{Immunohistochemical and immunofluorescence stainings}

Paraffin sections were deparaffinized, rehydrated and pretreated with antigen unmasking solution (Vector, Burlingame, CA, 8 min) followed by blocking with peroxidase blocking reagent (DAKO, Carpinteria, CA) and 3\% goat serum (Vector, ABC-Elite kit). The sections were then incubated overnight with the polyclonal goat antimouse adiponectin antibody (diluted 1:1000, RnD systems) followed by a biotinylated anti-goat secondary antibody for $30 \mathrm{~min}$ (Vector, ABC-Elite-Kit) and incubation with the avidin-peroxidase complex (Vector, Burlingame, CA). Colour was developed using the DAB substrate and sections were counterstained with hematoxylin (Vector, Burlingame, CA). Cryostat sections were fixed in acetone for $10 \mathrm{~min}$ and stained using the first and secondary antibodies as described above. Subsequently, the sections were incubated with streptavidinTexasRed (Vector, Burlingame, CA). Double stainings using CD31 and MECA-32 (BD Pharmingen) antibodies followed by a FITClabeled anti-rat antibody (DAKO) were performed. A negative control section was always included in which the primary antibody was substituted by the corresponding isotype control. The sections were analyzed without prior knowledge of treatment, and treatment response. The density of adiponectin ${ }^{+}$cells was evaluated by 
examining five high power fields, making a distinction between marked increase or few cells present.

\subsection{0. $N F-\kappa B$ activity}

$N F-\kappa B$ activation was measured using the EZ-Detect NF- $\kappa \mathrm{B}$ p65 Transcription Factor Kit (Pierce, Rockford, USA) strictly according to the manufacturer's instructions.

\subsection{Statistical analysis}

Results are expressed as the mean + SEM. Statistical analyses were performed using Student's $t$-test. A value of $P \leq 0.05$ was considered significant. Statistical analysis was performed using the GraphPadPrism software.

\section{Results}

\subsection{Adiponectin and its receptors are expressed in healthy liver tissue and regulated upon injection of ConA}

In healthy murine livers, we found a low but clearly detectable basal expression of adiponectin (Fig. 1(A)). It is noteworthy that special care was taken while preparing liver tissue for mRNA-isolation to avoid any contamination with mesenteric fat tissue. We further detected abundant expression of adiponectin receptor 1 and 2 (AdipoR1 and AdipoR2) mRNA, respectively (Fig. 1(B) and (C)). We next focussed on adiponectin expression under conditions of acute hepatic inflammation. Therefore, mice were injected with $15 \mathrm{mg} / \mathrm{kg}$ ConA. Real-time PCR revealed a $2.2 \pm 0.6$ fold increase of adiponectin mRNA expression within $8 \mathrm{~h}$ after challenge $(* P \leq 0.05)$. In contrast, acute hepatic inflammation reduced AdipoR1 and AdipoR2 mRNA expression levels to 30 and $60 \%$, respectively, when compared to healthy control livers $(* P \leq 0.05)$.

\subsection{Adiponectin is expressed by endothelial cells and hepatocytes in healthy and inflamed liver tissue}

To investigate whether the observed increase of adiponectin mRNA was accompanied by increased adiponectin protein levels, we performed immunohistochemical stainings of healthy as well as ConA-injected mouse livers. Adiponectin-expressing cells exhibit an endothelial cell-like phenotype (Fig. 2(A)). In line with the mRNA data, ConA-injection led to an increased number of adiponectin positive cells (Fig. 2(B) and (C)). To further define the adiponectin-producing cell type, we performed immunofluorescence double-stainings. Adiponectin positive cells, at least in part, stained positive for the endothelial cell markers CD31 (Fig. 2(E) and (F)) and MECA-32 (Fig. 2(G)).
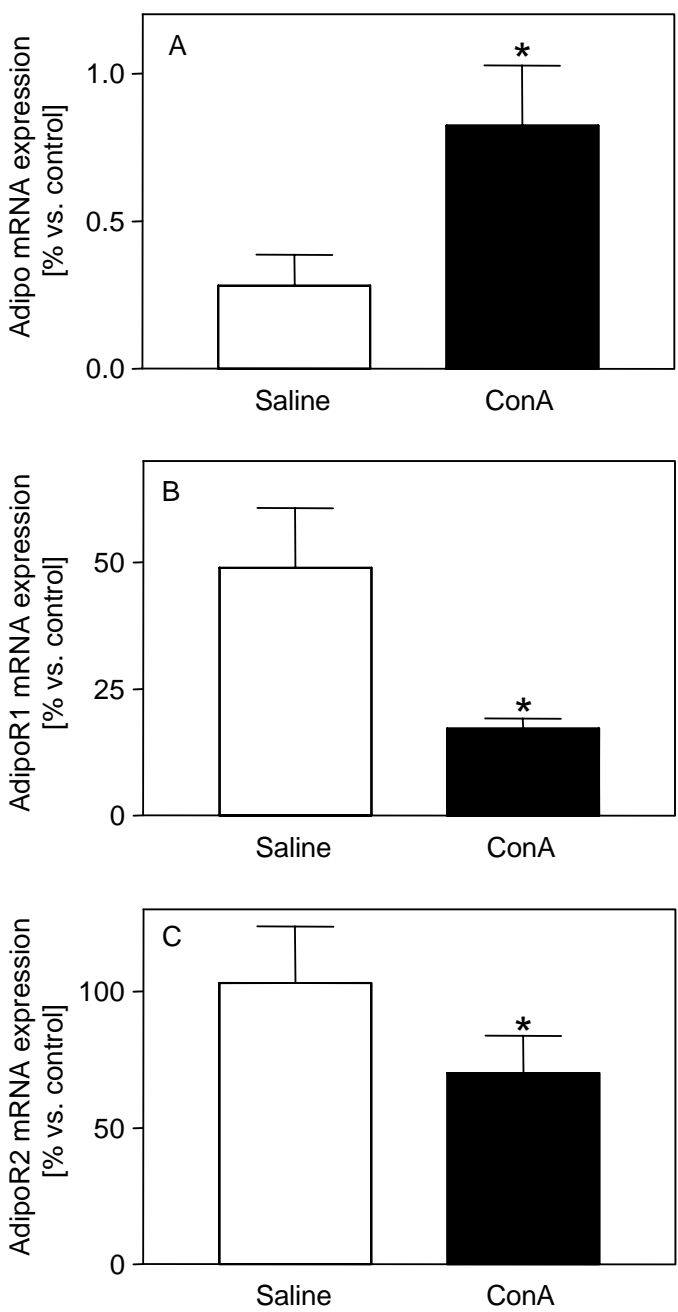

Fig. 1. Adiponectin mRNA is detectable in healthy murine liver tissue and is upregulated upon ConA injection. Mice were challenged either with saline or ConA $(15 \mathrm{mg} / \mathrm{kg}, n=5$ per group). (A) Hepatic adiponectin, (B) AdipoR1 and AdipoR2 (C) mRNA levels were determined by real-time PCR $8 \mathrm{~h}$ after ConA injection $(* P \leq 0.05)$.

Fig. 2(D) and (H) depict the corresponding isotype control.

\subsection{Detection of adiponectin $m R N A$ in isolated primary hepatocytes, hepatic sinusoidal endothelial cells, stellate cells and macrophages.}

To investigate in more detail the hepatic adiponectinexpressing cell-types we analysed adiponectin expression by quantitative RT-PCR in several hepatic cell types. According to our immunofluorescence and immunohistochemical stainings we detected adiponectin mRNA in isolated primary murine hepatic sinusoidal endothelial cells as well as in primary hepatic stellate cells (Fig. 3). Additionally, adiponectin mRNA was also found in CD11b-selected primary hepatic macrophages. No 

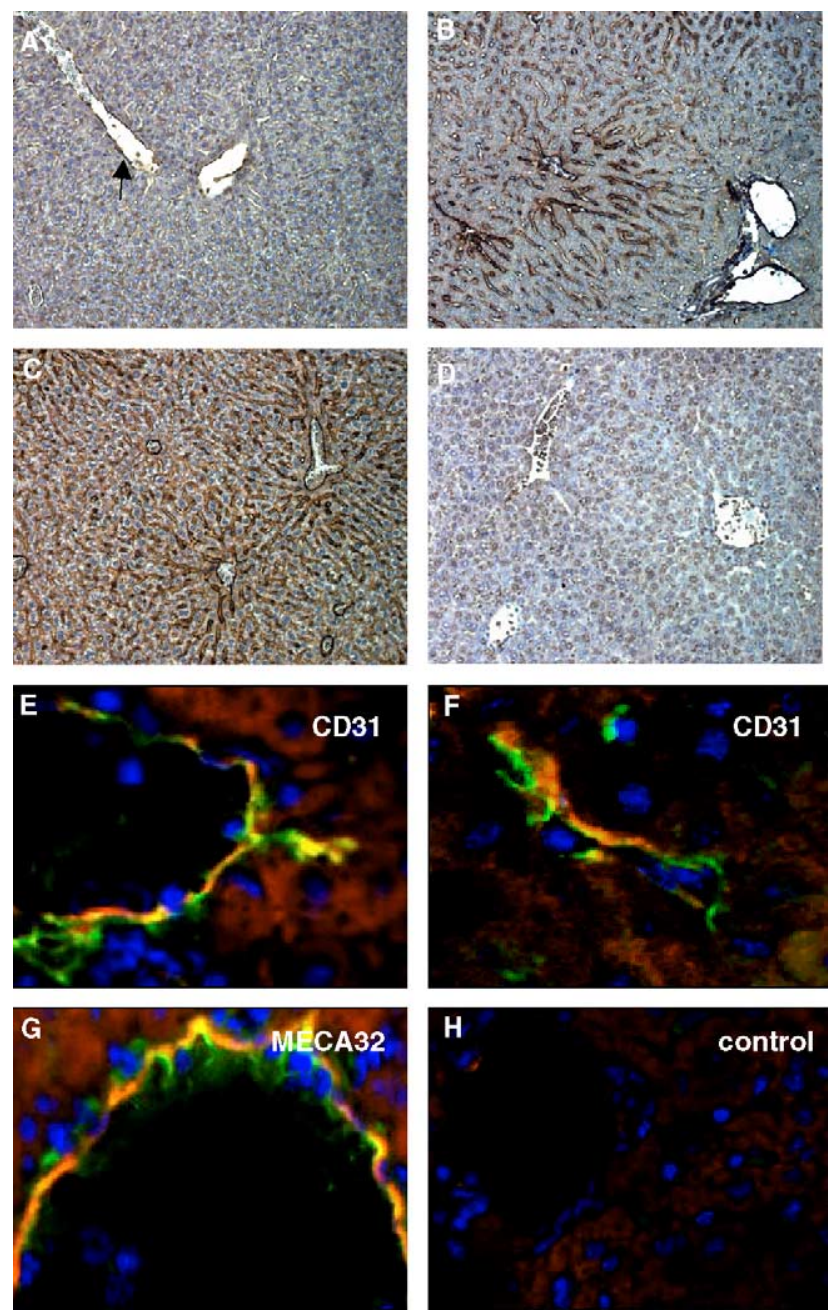

Fig. 2. Adiponectin is expressed by hepatic $\mathrm{CD31}^{+}, \mathrm{MECA}-32^{+}$ endothelial cells. Paraffinized tissue sections from ConA- and saline-injected animals were stained for adiponectin. (A) Basal hepatic adiponectin expression in healthy animals was detected in cells showing an endothelial phenotype (see arrow). (B and C) Increased adiponectin expression was detectable in ConA-injected mice (magnification $\times \mathbf{1 0 0}$ ). Immunofluorescence staining revealed that adiponectin-positive cells (red staining) co-expressed endothelial cell markers such as CD31 (E and F) and MECA-32 (G) (magnification $\times 400)$. ( $D$ and $H$ ) show the corresponding isotype control. A representative example out of three is shown.

adiponectin mRNA was detected in isolated primary mouse hepatocytes.

\subsection{Hepatoprotective effect of adiponectin in ConA-induced acute liver failure}

As adiponectin is known to decrease TNF- $\alpha$ expression in macrophages [7] and to increase the production of anti-inflammatory cytokines we next aimed to analyze the impact of adiponectin on TNFdependent acute liver failure. Therefore, we either

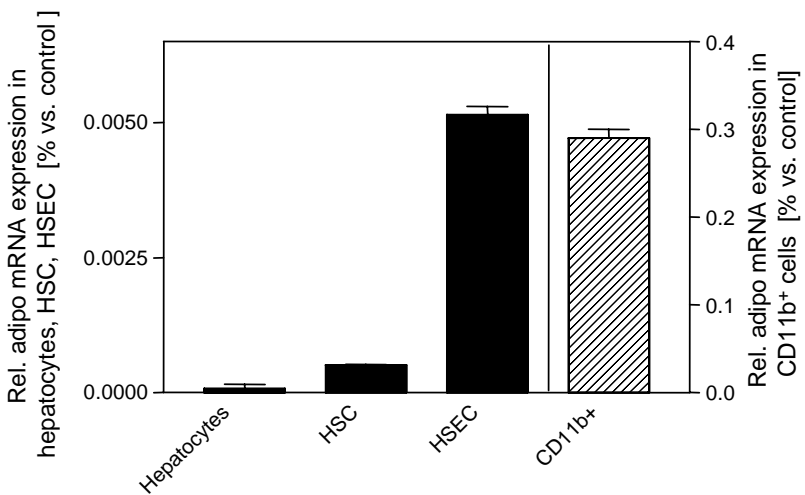

Fig. 3. Detection of adiponectin mRNA in isolated primary hepatic stellate cells, hepatic sinusoidal endothelial cells, and liver macrophages. mRNA was obtained from isolated primary hepatocytes, hepatic stellate cells, hepatic sinusoidal endothelial cells, and CD11bselected macrophages and adiponectin expression levels were determined by real-time PCR. Tissue expression levels are given relative to a positive control as described in the Section 2.

injected $6 \mathrm{mg} / \mathrm{kg}$ recombinant murine adiponectin or solvent $12 \mathrm{~h}$ prior to application of ConA. Adiponectin pre-treatment significantly decreased the ConA-induced hepatotoxicity as shown by release of liver enzymes (Fig. 4(A), *P $\leq 0.05)$. These data were corroborated by a lower number of TUNEL-positive apoptotic hepatic cells (Fig. 4(B)). Using cytometric bead arrays and ELISA we were not able to detect significant changes in plasma levels of TNF- $\alpha$ and IL-6 (Fig. 4(C) and (D)). However, IL-10 production was significantly increased in adiponectin-treated mice (Fig. 4(E), $* P \leq 0.05$ ). Comparable results were obtained for hepatic mRNA regulation as determined by quantitative RT-PCR: Adiponectin did not change hepatic TNF- $\alpha$ mRNA expression, whereas IL-10 mRNA expression was increased by adiponectin (data not shown). Thus, the protective effect of adiponectin administration in this model does not appear to be mediated by reduced intrahepatic TNF- $\alpha$ levels.

\subsection{The hepatoprotective effect of adiponectin is partially mediated by IL-10}

We next tested whether the induction of IL-10 is causally involved in adiponectin-induced hepatoprotection. Therefore, animals were injected with a neutralizing antibody directed against IL-10 prior to either adiponectin- or ConAinjection. In line with previous reports, neutralisation of IL10 resulted in increased ConA-induced TNF- $\alpha$ production (Fig. 5(A), $P<0.05)$. Notably, anti-IL-10 treatment in part abrogated the hepatoprotective effects of adiponectin as shown by liver enzyme release: in contrast to the significant reduction of ALT release by adiponectin, ALT levels in the anti-IL10 pre-treated group did not statistically differ from the saline/ConA control group (Fig. 5(B), *P $\leq 0.05$ for 


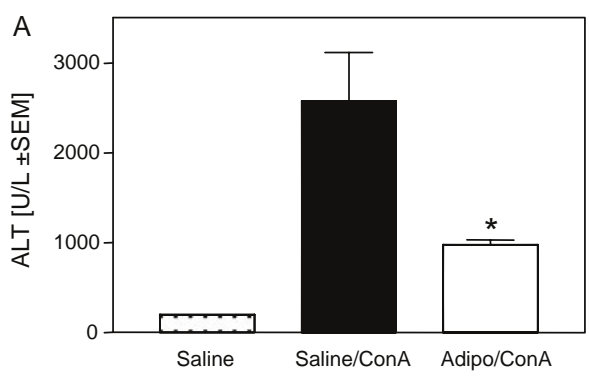

B
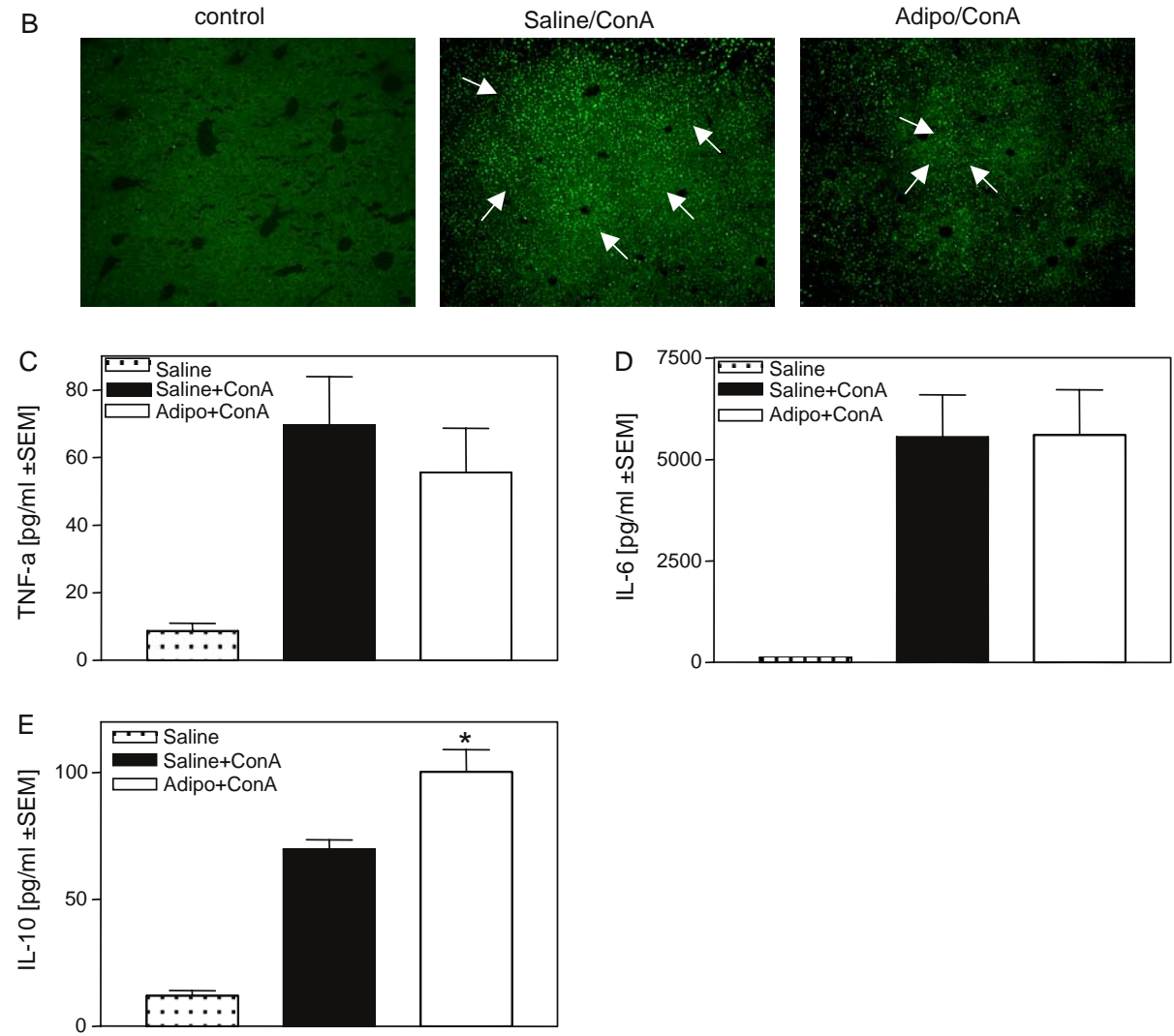

Fig. 4. Hepatoprotective effect of adiponectin in ConA-induced acute liver failure. Either adiponectin or saline-pre-treated mice were challenged with Con A $(15 \mathrm{mg} / \mathrm{kg}, n=8$ per group). (A) Plasma ALT-levels were determined $8 \mathrm{~h}$ after Con A injection (*P $\leq 0.05)$. (B) Adiponectin markedly reduced the induction of apoptosis detected by TUNEL-staining $8 \mathrm{~h}$ after challenge. A representative example from each group is shown (magnification $\times 100$ ). (C) TNF- $\alpha$, and (D) IL-6 plasma levels were determined $2 \mathrm{~h}$ and (E) IL-10 plasma levels $(* P<0.05)$ were determined $8 \mathrm{~h}$ after ConA injection by ELISA.

Adipo/ConA vs. saline/ConA and $P>0.05$ for $\alpha$-IL10/ Adipo/ConA vs. saline/ConA).

\subsection{The ConA-mediated hepatic $N F-\kappa B$ activation is significantly down-regulated by adiponectin}

$N F-\kappa B$ represents a key transcription factor for the regulation of ConA-induced cytokine gene expression [8]. We, therefore, analyzed the activation of NF- $\mathrm{B}$ p65 upon ConA-injection in mice pre-treated with either adiponectin or solvent. Indeed, ConA-mediated activation of NF- $\kappa \mathrm{B}$ was significantly inhibited by adiponectin pre-exposure (Fig. 6, $* P \leq 0.05)$.

\section{Discussion}

Adiponectin, the most abundant adipokine in humans, has recently been shown to exert potent anti-inflammatory effects in humans and in mice. To date, the regulation of adiponectin has been nearly exclusively studied in the adipocyte, which has been considered the unique site of adiponectin production under physiological conditions [9]. However, two recent studies reported that adiponectin gene 

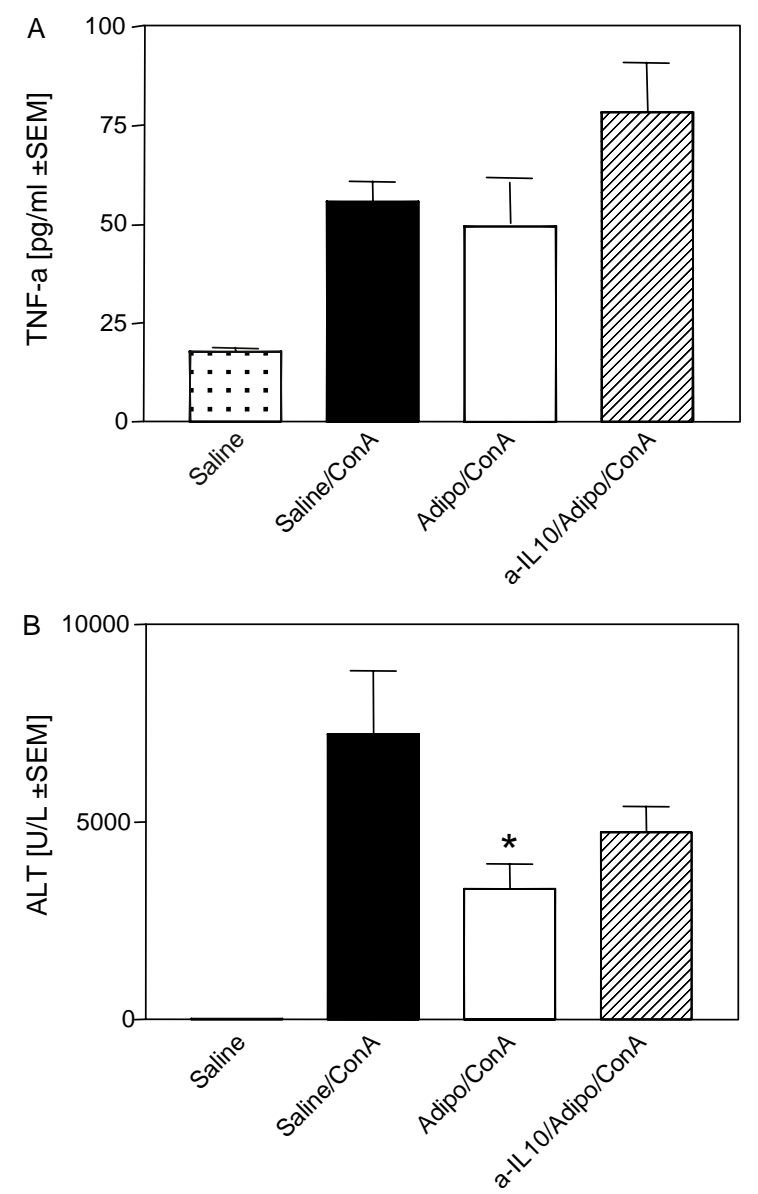

Fig. 5. The hepatoprotective effect of adiponectin is partially mediated by IL-10. Either adiponectin/ConA or saline/ConA treated mice were pre-treated with a neutralizing IL-10 antibody $(3 \mathrm{mg} / \mathrm{mouse}, n=4$ per group) followed by ConA injection. (A) Plasma TNF-levels were determined $2 \mathrm{~h}$ after ConA injection by ELISA (B) Plasma ALT-levels were determined $8 \mathrm{~h}$ after $\mathrm{ConA}$ injection $(* P<0.05)$.

expression is inducable in human myotubes upon stimulation with pro-inflammatory cytokines, such as IFN- $\gamma$ and TNF- $\alpha[5,6]$. In the current study, we show that apart from high expression in adipose tissue, adiponectin is also expressed in non-inflammatory murine liver tissue. Real-

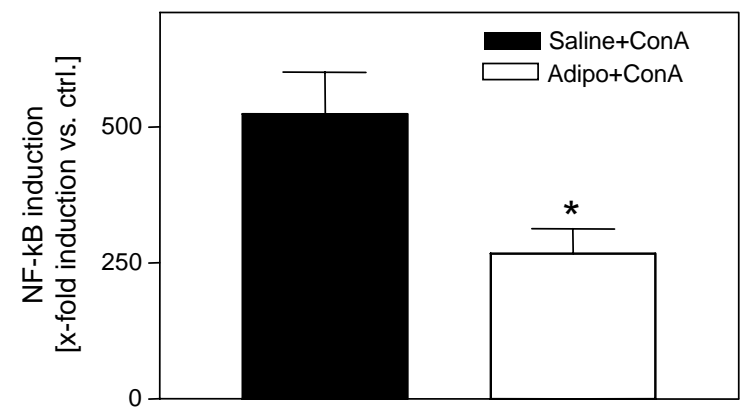

Fig. 6. Down-regulation of ConA-mediated hepatic NF- $\mathrm{B}$ activation by adiponectin. Whole liver lysates were prepared from either adiponectin/ConA- or saline/ConA-treated mice and NF- $\kappa B$ p65 activation was determined using a chemiluminescent assay $(n=4$, $* \boldsymbol{P} \leq \mathbf{0 . 0 5})$. time PCR revealed that adiponectin mRNA is produced in several cells types i.e. isolated primary murine liver sinusoidal endothelial cells, hepatic stellate cells and liver macrophages. Induction of acute hepatic inflammation by ConA led to a significant up-regulation of adiponectin. These data are corroborated by a recent report from YodaMurakami and co-workers showing an increase of adiponectin in inflamed liver tissue induced by injection of $\mathrm{CCl}_{4}$ [10]. ConA-induced hepatic inflammation is characterized by highly elevated systemic as well as hepatic TNF- $\alpha$ levels [11]. In addition, adiponectin has been shown to be a major antagonist of TNF- $\alpha$ production in several cell types [7,12]. Thus, its induction under conditions of acute inflammation within hepatic tissue might counteract the pro-inflammatory actions of TNF- $\alpha$ thereby forming a negative feedback loop attempting to counterbalance hepatic inflammation in vivo. In line with this idea, Maeda and co-workers recently demonstrated that adiponectin-deficient mice revealed high levels of TNF- $\alpha$ mRNA in adipose tissue as well as high plasma TNF- $\alpha$ concentration [13]. Moreover, adiponectintreatment protects KK-Ay obese mice, which are characterized by low circulating adiponectin levels from TNF- $\alpha$ mediated acute hepatic failure induced by LPS [12]. The authors nicely demonstrate that the protective effect of adiponectin in KK-Ay obese mice is primarily mediated by down-regulating the sensitivity of hepatocytes to TNFinduced apoptosis, without affecting cytokine levels in these animals.

We extend these findings by showing that application of adiponectin to $\mathrm{BALB} / \mathrm{c}$ mice markedly attenuated liver inflammation induced by ConA. In line with the findings from Sennello and co-workers [14], we were not able to detect a down-regulation of either circulating or hepatic TNF- $\alpha$ levels. Therefore, adiponectin probably exerts its hepatoprotective effects in the model of acute liver inflammation induced by ConA by a mechanism independent of TNF- $\alpha$ down-regulation. We and others recently described an anti-inflammatory role of adiponectin on in vitro cultured myeloid cells $[3,4,7]$. Pre-treatment of monocytes and monocyte-derived cells (i.e. macrophages and dendritic cells) with adiponectin impairs the LPSinduced production of pro-inflammatory cytokines. This effect is paralleled by an increased production of antiinflammatory cytokines, such as IL-10. Accordingly, adiponectin pre-treatment significantly increased IL-10 production in ConA-injected animals. Blocking IL-10 by neutralizing antibodies partially reversed the beneficial effects of adiponectin in vivo. Thus, induction of IL-10 seems to play at least a partial role in the protective effects of adiponectin. The TNF- $\alpha / \mathrm{NF}-\kappa \mathrm{B}$ pathway plays a critical role in inflammatory signaling processes. Adiponectin was demonstrated to suppress TNF- $\alpha$-induced activation of nuclear factor- $\kappa \mathrm{B}(\mathrm{NF}-\kappa \mathrm{B})$ in endothelial cells, therefore, acting as an endogenous biologically relevant modulator of endothelial cell responses to pro-inflammatory stimuli [15]. Moreover, adiponectin has been shown to inhibit 
LPS-induced activation of $\mathrm{NF}-\kappa \mathrm{B}$ in adipocyte cell lines. According to this data, adiponectin inhibited hepatic NF- $\kappa \mathrm{B}$ signaling in ConA-induced acute liver failure. Recent reports demonstrated that hepatoprotective and antiapoptotic molecules such as $S$-Adenosylmethionine and PGE1 may reduce $\mathrm{NF}-\kappa \mathrm{B}$ activation in hepatocytes $[16,17]$. However, further signaling pathways being involved in the anti-apoptotic effects of adiponectin in hepatocytes remain to be elucidated. The activation of the AMP-kinase for example has been shown to be involved in protection from mitogen-deprivation induced cell death in HUVEC [18].

In conclusion, our data clearly show that under inflammatory conditions adiponectin can be produced by other cell types, i.e. by hepatic endothelial and stellate cells. Application of adiponectin decreases hepatic injury, which suggests that adiponectin may play an important role in the control and limitation of inflammatory processes in the liver.

\section{Acknowledgements}

This work was supported by the Austrian Science Fund FWF (grant P17447 to H.T.).

\section{References}

[1] Scherer PE, Williams S, Fogliano M, Baldini G, Lodish HF. A novel serum protein similar to $\mathrm{C} 1 \mathrm{q}$, produced exclusively in adipocytes. J Biol Chem 1995;270:26746-26749.

[2] Yamauchi T, Hara K, Kubota N, Terauchi Y, Tobe K, Froguel P, et al. Dual roles of adiponectin/Acrp30 in vivo as an anti-diabetic and antiatherogenic adipokine. Curr Drug Targets Immune Endocrinol Metabol Disord 2003;3:243-254.

[3] Wolf AM, Wolf D, Rumpold H, Enrich B, Tilg H. Adiponectin induces the anti-inflammatory cytokines IL-10 and IL-1RA in human leukocytes. Biochem Biophys Res Commun 2004;323: 630-635.

[4] Wulster-Radcliffe MC, Ajuwon KM, Wang J, Christian JA, Spurlock ME. Adiponectin differentially regulates cytokines in porcine macrophages. Biochem Biophys Res Commun 2004;316: 924-929.
[5] Delaigle AM, Jonas JC, Bauche IB, Cornu O, Brichard SM. Induction of adiponectin in skeletal muscle by inflammatory cytokines: in vivo and in vitro studies. Endocrinology 2004;145: 5589-5597.

[6] Staiger H, Kausch C, Guirguis A, Weisser M, Maerker E, Stumvoll M, et al. Induction of adiponectin gene expression in human myotubes by an adiponectin-containing HEK293 cell culture supernatant. Diabetologia 2003;46:956-960.

[7] Yokota T, Oritani K, Takahashi I, Ishikawa J, Matsuyama A, Ouchi N, et al. Adiponectin, a new member of the family of soluble defense collagens, negatively regulates the growth of myelomonocytic progenitors and the functions of macrophages. Blood 2000;96: $1723-1732$.

[8] Tiegs G, Hentschel J, Wendel A. A T cell-dependent experimental liver injury in mice inducible by concanavalin A. J Clin Invest 1992; 90:196-203.

[9] Havel PJ. Control of energy homeostasis and insulin action by adipocyte hormones: leptin, acylation stimulating protein, and adiponectin. Curr Opin Lipidol 2002;13:51-59.

[10] Yoda-Murakami M, Taniguchi M, Takahashi K, Kawamata S, Saito K, Choi-Miura $\mathrm{NH}$, et al. Change in expression of GBP28/adiponectin in carbon tetrachloride-administrated mouse liver. Biochem Biophys Res Commun 2001;285:372-377.

[11] Gantner F, Leist M, Lohse AW, Germann PG, Tiegs G. Concanavalin A-induced T-cell-mediated hepatic injury in mice: the role of tumor necrosis factor. Hepatology 1995;21:190-198.

[12] Masaki T, Chiba S, Tatsukawa H, Yasuda T, Noguchi H, Seike M, et al. Adiponectin protects LPS-induced liver injury through modulation of TNF-alpha in KK-Ay obese mice. Hepatology 2004; 40:177-184.

[13] Maeda N, Shimomura I, Kishida K, Nishizawa H, Matsuda M, Nagaretani $\mathrm{H}$, et al. Diet-induced insulin resistance in mice lacking adiponectin/ACRP30. Nat Med 2002;8:731-737.

[14] Sennello JA, Fayad R, Morris AM, Eckel RH, Asilmaz E, Montez J, et al. Regulation of $\mathrm{T}$ cell-mediated hepatic inflammation by adiponectin and leptin. Endocrinology 2005;146:2157-2164.

[15] Ouchi N, Kihara S, Arita Y, Okamoto Y, Maeda K, Kuriyama H, et al. Adiponectin, an adipocyte-derived plasma protein, inhibits endothelial NF-kappaB signaling through a cAMP-dependent pathway. Circulation 2000;102:1296-1301.

[16] Majano PL, Garcia-Monzon C, Garcia-Trevijano ER, Corrales FJ, Camara J, Ortiz P, et al. S-Adenosylmethionine modulates inducible nitric oxide synthase gene expression in rat liver and isolated hepatocytes. J Hepatol 2001;35:692-699.

[17] Siendones E, Fouad D, Diaz-Guerra MJ, de la MM, Bosca L, Muntane J. PGE1-induced NO reduces apoptosis by D-galactosamine through attenuation of NF-kappaB and NOS-2 expression in rat hepatocytes. Hepatology 2004;40:1295-1303.

[18] Kobayashi H, Ouchi N, Kihara S, Walsh K, Kumada M, Abe Y, et al. Selective suppression of endothelial cell apoptosis by the high molecular weight form of adiponectin. Circ Res 2004;94:e27-e31. 